\title{
Free caloron gas in high temperature quenched QCD
}

\author{
Reka A. Vig \\ University of Debrecen \\ E-mail: vig.reka@atomki.mta.hu \\ Tamas G. Kovacs* \\ Institute for Nuclear Research, Debrecen \\ E-mail: kgteatomki.mta.hu
}

\begin{abstract}
Across the finite temperature transition to the quark-gluon plasma, the QCD topological susceptibility decreases sharply. Thus in the high temperature phase the remaining topological objects (possibly calorons) form a weakly interacting dilute gas. The overlap Dirac operator, through its exact zero modes, allows one to measure the net topological charge. We show that separately the number of positively and negatively charged topological objects can also be extracted from the low end of the overlap Dirac spectrum. We find that slightly above the phase transition their number distributions are already consistent with an ideal gas of non-interacting topological objects.
\end{abstract}

37th International Symposium on Lattice Field Theory - Lattice2019

16-22 June 2019

Wuhan, China

${ }^{*}$ Speaker. 


\section{Introduction}

It is well known that the finite temperature crossover of QCD is accompanied by rapid changes in the Polyakov loop and the quark condensate, the order parameters of confinement and chiral symmetry. In particular, as the (approximate) chiral symmetry, spontaneously broken at zero temperature, gets restored above the crossover, the quark condensate drops sharply. Since the Banks-Casher [1] relation connects the quark condensate to the spectral density of the Dirac operator around zero, the latter also falls sharply as the system crosses into the high temperature quark-gluon plasma phase. According to the traditional picture of chiral restoration, as shown in the schematic plots of Fig. 1, the spectral density at zero becomes vanishingly small in the high temperature phase.
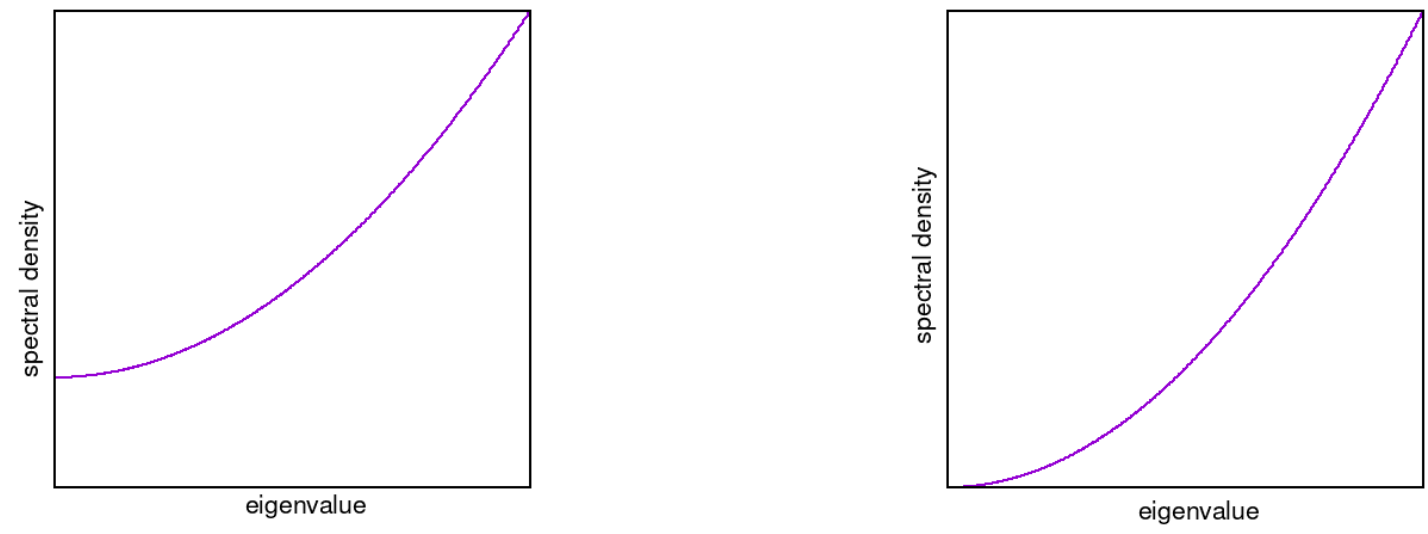

Figure 1: Schematic depiction of the spectral density of the Dirac operator around zero virtuality at low temperature (left panel) and high temperature (right panel). According to the Banks-Casher relation the spectral density at zero is the order parameter of chiral symmetry.

However, in reality the behavior of the spectral density across the transition might be more complicated. Based on a study of the spectrum of the overlap Dirac operator in quenched gauge backgrounds, it was already suggested a long time ago that just above the transition temperature the spectral density at zero does not vanish, but develops a "spike" [2]. Light dynamical fermions are expected to suppress low Dirac eigenvalues, as those eigenvalues contribute small factors to the Dirac determinant. Thus the excess of small eigenvalues and the resulting spike in the spectrum was initially thought to be a quenched artifact. However, more recently it was suggested that even light quarks cannot completely eliminate the spectral spike, at least as long as the quarks are not exactly massless [3,4]. Already the authors of [2] raised the possibility that this excess of low Dirac eigenvalues may be connected to topological objects in the gauge field, and the distribution of the number of eigenvalues in the spike supported this assumption.

In the present paper we study this question in detail using large ensembles of quenched gauge field configurations in the high temperature phase and the overlap Dirac operator. Our main result is that around zero virtuality in the Dirac spectrum there is a clearly separated region that is very likely to be connected to topological objects in the gauge field. Above $T_{c}$ the Dirac spectrum can thus be 
used to identify not only the net topological charge through zero modes, but also the total number of topological objects. This enables us to examine their interactions, and in this quenched study we do not find any trace of interactions among topological objects, to a very good approximation they can be described as a dilute non-interacting gas.

\section{Topology and the zero mode zone}

In this section we briefly discuss how the lowest part of the Dirac spectrum can be connected to topological fluctuations of the gauge field. It is an old idea that the nonzero density of Dirac eigenvalues around zero that is needed for the spontaneous breaking of chiral symmetry, is provided by mixing approximate zero modes of a densely packed medium of instantons and antiinstantons, called the instanton liquid (see [5] for a review). The mixing of topological zero modes can be easily understood by considering an instanton antiinstanton pair separated by a distance much larger than their size. If the separation were infinite, the Dirac operator would have two opposite chirality zero modes. However, an arbitrarily small perturbation, due to the two objects being a finite distance apart, will split the degeneracy of the zero modes, resulting in two complex conjugate eigenvalues of very small magnitude. Their magnitude, i.e. their splitting from zero is mainly controlled by the distance of the topological objects; the closer they are, the larger the perturbation of the zero modes and the larger the splitting will be.

Unfortunately, in QCD at zero temperature, even if there are instantons, they are too closely packed for this picture to be applicable in a straightforward way. In particular, it is not possible to identify those small non-zero eigenvalues in the spectrum that are connected to topology. This is because the spectral density is monotonically rising everywhere, and the lowest part of the spectrum, termed the zero mode zone (ZMZ) is smoothly connected to the bulk of the spectrum. The situation, however, changes drastically as the system crosses over into the high temperature, chirally restored phase. As the temperature increases and the (Euclidean) temporal size of the system shrinks, bigger instantons are squeezed out and fluctuations of the topological charge fall sharply, the instanton liquid turns into a dilute gas of finite temperature topological objects, calorons. As the typical distance between topological objects increases, the splitting of the approximate zero modes becomes ever smaller and these small Dirac eigenvalues are expected to be present in the spectrum with a finite volume density, their density corresponding to that of the topological objects surviving at the given temperature. The question is whether these small eigenvalues of topological origin can be identified in the spectrum and separated from the bulk modes, the ones not related to topology.

\section{Identification of the $\mathrm{ZMZ}$ above $T_{c}$}

In this section, based on numerical evidence we argue that the accumulation of small Dirac eigenvalues above $T_{c}$ can indeed be connected to topology, and we also demonstrate that the ZMZ can be clearly identified in the spectrum of the overlap Dirac operator. In Fig. 2 we show the spectral density of the overlap operator on ensembles of quenched $S U(3)$ gauge field configurations on lattices of temporal size $N_{t}=6$ and different spatial volumes. The temperature is $T=1.04 T_{c}$, just above the transition, which in the quenched case, unlike in QCD with physical quarks, is 


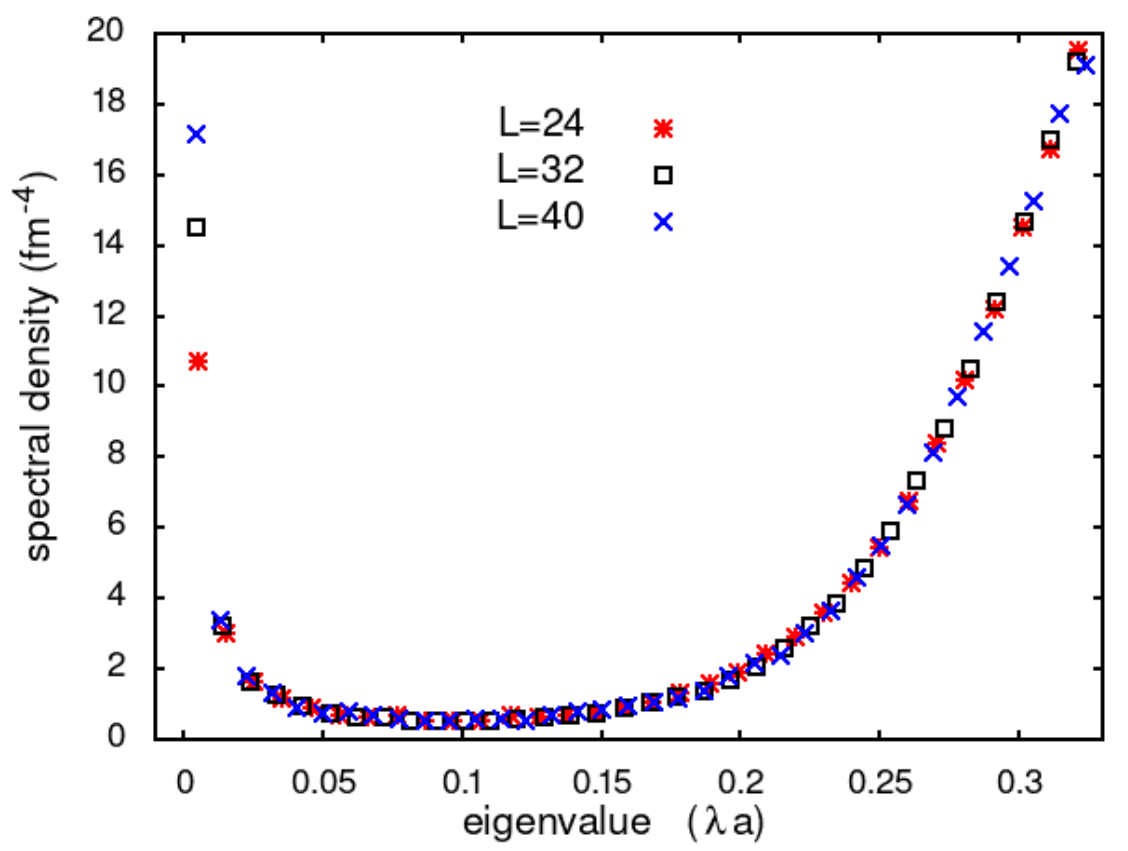

Figure 2: The spectral density of the overlap Dirac operator in quenched gauge field backgrounds at $T=1.04 T_{c}$. The temporal extension of the lattice is $N_{t}=6$ and the different symbols correspond to spatial linear extensions $L=24,32,40$.

a genuine phase transition. The most striking feature of the spectral density is that instead of smoothly and monotonically vanishing at $\lambda=0$, it has a spike there. We emphasize that the spike seen in the figure is solely due to near zero modes, as the exact zero modes have been removed from the spectral density.

It is tempting to identify the spike with the zero mode zone, i.e. the mixing near zero modes connected to fluctuations of the gauge field topological charge. How could this assumption be checked? One possibility is to check whether the average number of eigenmodes in the spike and their distribution are consistent with the fluctuations of the net topological charge, as given by the topological susceptibility.

Since we computed overlap spectra on these configurations, we have the number of exact zero modes, i.e. $n_{\mathrm{i}}-n_{\mathrm{a}}$, the difference of the number of instantons and antiinstantons ${ }^{1}$, configuration by configuration. This in turn determines the topological charge $Q$ and the topological susceptibility as

$$
\chi=\frac{1}{V}\left\langle Q^{2}\right\rangle=\frac{1}{V}\left\langle\left(n_{\mathrm{i}}-n_{\mathrm{a}}\right)^{2}\right\rangle,
$$

where $\langle$.$\rangle denotes averaging with the path integral measure and V$ is the space-time volume. If the eigenmodes in the spike of the spectral density are the ones corresponding to topological charge,

\footnotetext{
${ }^{1}$ Strictly speaking, at finite temperature the exact solutions of the Euclidean equations of motion for the gauge field are calorons. Nevertheless, we will freely use the words instanton and caloron for approximate solutions at high temperature.
} 
then their number $n_{\text {spike }}$ together with that of the exact zero modes is equal to the total number of topological objects, i.e.

$$
n_{\mathrm{i}}+n_{\mathrm{a}}=n_{\text {spike }}+|Q| .
$$

These numbers and their distributions are in principle independent and complicated variables, determined by the dynamics of the system. However, they are all connected in a simple way if topological objects occur statistically independently. Fortunately, at high temperature topological fluctuation are suppressed, calorons are expected to form a dilute gas and the interaction among topological objects becomes smaller. If the interaction is completely neglected then all the statistical properties of the distribution of topological objects are encoded in a single parameter, for example the topological susceptibility $\chi$. In this case it is easy to show that the distribution of the number of instantons and antiinstantons in the gauge configurations are given by two independent and identical Poisson distributions with expectation $\frac{1}{2} V \chi$. It is also not hard to show that the probabilities of the different charge sectors $Q$ are given by

$$
P_{\mathrm{Q}}=\mathrm{e}^{-\chi V} I_{\mathrm{Q}}(\chi V)
$$

where $I_{\mathrm{Q}}$ are the modified Bessel functions of order $Q$.

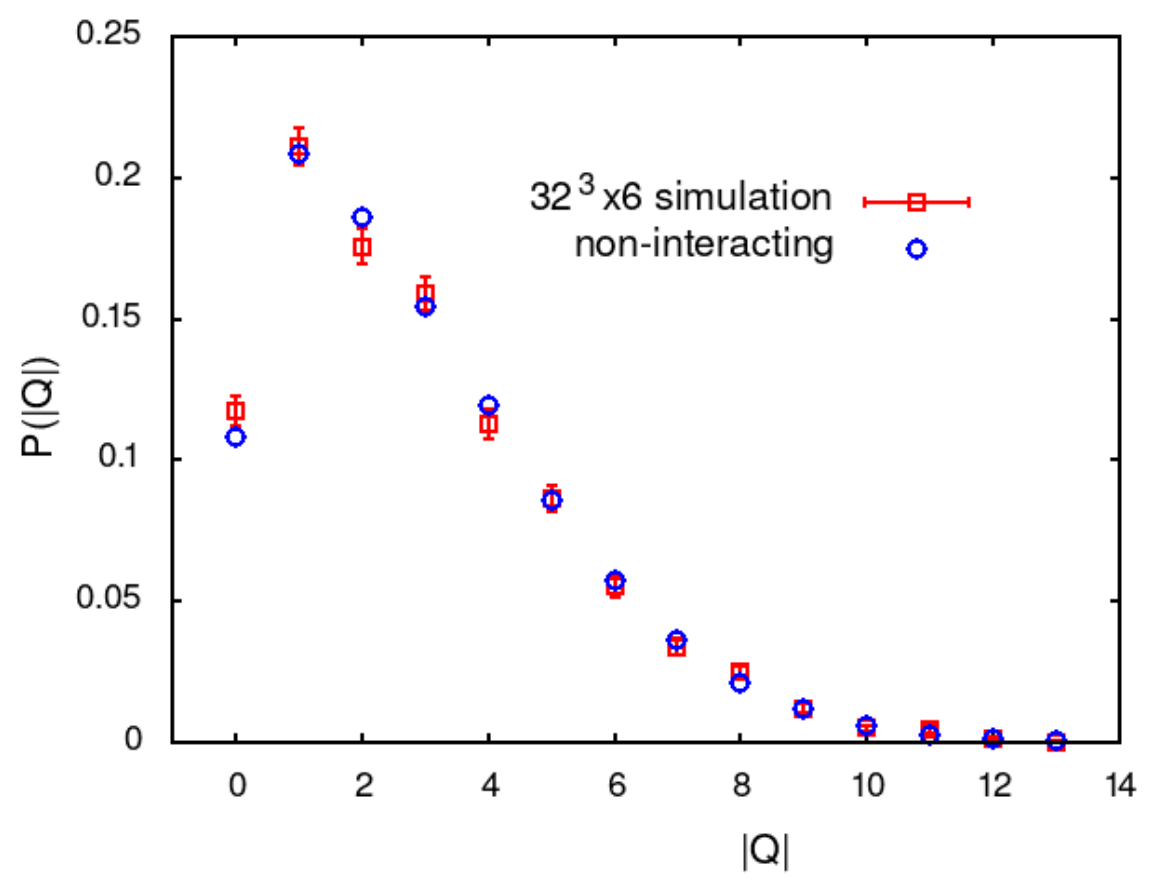

Figure 3: The probability of the different topological charge sectors obtained from overlap zero modes on a set of $380032^{3} \times 6$ lattice configurations at $T=1.04 T_{\mathrm{c}}$ (squares). Due to the expected symmetry, the probabilities of the charge sectors $Q$ and $-Q$ were added. The circles show the expected probabilities in a free instanton gas with the same topological susceptibility.

To test whether the caloron gas is non-interacting, in Fig. 3 we compare the numerically obtained charge distribution with the distribution of Eq. (3.3) expected for free calorons. The parameter $\chi$ of the latter distribution was set equal to the numerically obtained topological susceptibility, so 
the comparison effectively amounts to a one-parameter fit. Surprisingly, even at this relatively low temperature, $T=1.04 T_{\mathrm{c}}$, no significant discrepancy can be observed between the non-interacting instanton model and the lattice data.

Encouraged by the good agreement of the lattice data and the free instanton gas description, we can extend our study to the full zero mode zone, including also the near zero modes. Again, based on the free instanton gas, it is easy to see that the distribution of $n_{\mathrm{i}}+n_{\mathrm{a}}$ is Poisson, with expectation $V \chi$. To be able to check this on the lattice data, we have to fix an additional parameter, a cut in the spectrum, $\lambda_{\mathrm{zmz}}$, below which eigenvalues are considered to belong to the zero mode zone. The most natural way to fix $\lambda_{\mathrm{zmz}}$ is to require that

$$
\left\langle n_{\mathrm{i}}+n_{\mathrm{a}}\right\rangle=V \chi
$$

on the given ensemble of gauge configurations, where $\chi$ is again the topological susceptibility obtained by counting zero modes. In a free instanton gas eq. (3.4) holds exactly and for a lattice ensemble of configurations, by definition $\left\langle n_{\mathrm{i}}+n_{\mathrm{a}}\right\rangle$ is the average number of eigenvalues with $|\lambda|<$ $\lambda_{\mathrm{zmz}}$, including exact zero modes.

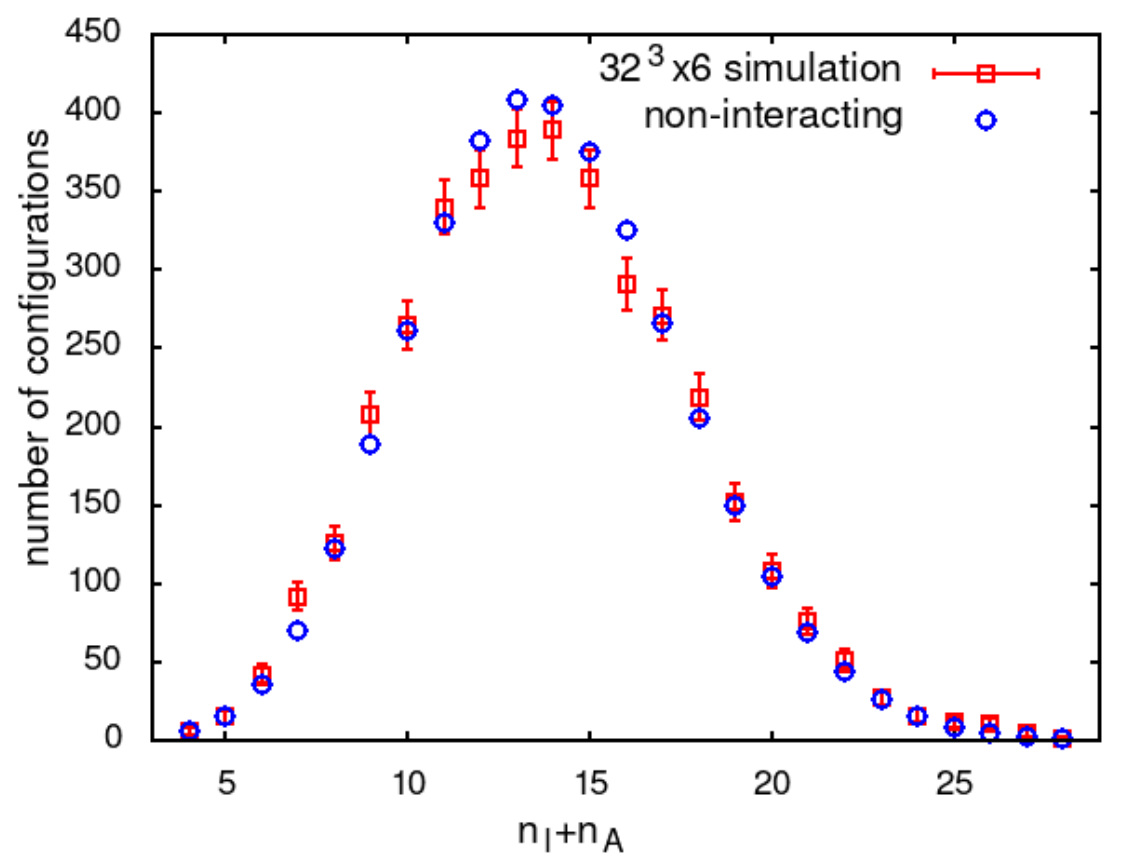

Figure 4: The occurrence of different numbers of topological objects (instantons plus antiinstantons) obtained from overlap near zero modes on a set of $380032^{3} \times 6$ lattice configurations at $T=1.04 T_{\mathrm{c}}$ (squares) compared to the numbers expected for an ideal gas of calorons (circles). The cut for the counted near zero modes was set to $\lambda a=0.092$, consistently with the susceptibility, as explained in the text.

Fixing $\lambda_{\mathrm{zmz}}$ in this way, we compare the numerically obtained and the expected distribution in Fig. 4, and we find good agreement again. The distributions for the two other spatial lattice volumes, $24^{3}$ and $40^{3}$ look similar and neither of them shows any significant deviation from the distributions expected in a free caloron gas. The values of $\lambda_{\mathrm{zmz}}$ determined on the three ensembles 
are consistent with one another and their combined average is $\lambda_{\mathrm{zmz}} a=0.09 \pm 0.01$. As a further consistency check we can see in Fig. 2 that this point in the spectrum roughly coincides with the minimum of the spectral density between the spike at zero and the bulk. This indicates that the modes of topological origin can indeed be separated from the bulk of the spectrum. We also expect this separation to become more pronounced in the continuum limit and also at higher temperatures.

Another interesting feature of the spectral density in Fig. 2 is that for very small values of $\lambda$ (most conspicuously in the first bin) there are unusually large corrections to the proper volume scaling of the spectral density. However, as a more detailed analysis reveals, the total number of topological modes scales properly with the volume. The discrepancy in the figure is caused by the fact that the topological modes corresponding to the net charge occur exactly at zero, but the density of these zero modes vanishes with $1 / \sqrt{V}$ and they will not contribute to the spectral density in the thermodynamic limit. However, at these volumes their contribution is still seen, resulting also in significantly smaller spectral densities at the smallest $\lambda$ values.

\section{Conclusions}

In the present paper we gave strong indications that in quenched QCD already a bit above $T_{\mathrm{c}}$ the topology related near zero modes of the overlap Dirac operator can be separated from the bulk of the spectrum. Counting these modes, as well as the exact zero modes configuration by configuration, we found the resulting distributions to be consistent with the ones expected for a free caloron gas. The present study was based on the quenched approximation, where the quark determinant is neglected. However, the Dirac determinant with light quarks is expected to be sensitive to small Dirac eigenvalues and through them also to topology which in turn could result in interactions among topological objects. Therefore, the most interesting extension of this work would be to repeat the study with the inclusion of light dynamical quarks. Unfortunately, the presence or absence of quark induced interactions among topological objects could crucially depend on the chiral properties of the Dirac operator, i.e. how well it can resolve zero and near zero modes. It would also be interesting to see how these topological modes are related to the possible new phases of QCD discussed in Ref. [6].

\section{References}

[1] T. Banks and A. Casher, Nucl. Phys. B 169, 103 (1980). doi:10.1016/0550-3213(80)90255-2.

[2] R. G. Edwards, U. M. Heller, J. E. Kiskis and R. Narayanan, Phys. Rev. D 61, 074504 (2000) doi:10.1103/PhysRevD.61.074504 [hep-lat/9910041].

[3] A. Alexandru and I. Horváth, Phys. Rev. D 92, no. 4, 045038 (2015) doi:10.1103/PhysRevD.92.045038 [arXiv:1502.07732 [hep-lat]].

[4] V. Dick, F. Karsch, E. Laermann, S. Mukherjee and S. Sharma, Phys. Rev. D 91, no. 9, 094504 (2015) doi:10.1103/PhysRevD.91.094504 [arXiv:1502.06190 [hep-lat]].

[5] T. Schäfer and E. V. Shuryak, Rev. Mod. Phys. 70, 323 (1998) doi:10.1103/RevModPhys.70.323 [hep-ph/9610451].

[6] A. Alexandru and I. Horvath, arXiv:1906.08047 [hep-lat]; see also I. Horváth's contribution to this conference. 